\title{
Prognostic value of natriuretic peptides in severe trauma patients with multiple organ dysfunction syndrome
}

\author{
NAN LI, ZHI SONG, JING WANG, YUE TENG, YAN CUI, HONGXU JIN and YAN GAO \\ Department of Emergency Medicine, General Hospital of Shenyang Military Region, \\ Shenyang, Liaoning 110016, P.R. China
}

Received August 1, 2014; Accepted April 7, 2015

DOI: 10.3892/etm.2015.2570

\begin{abstract}
The aim of the present study was to evaluate the prognostic values of the N-terminal peptide of pro-atrial natriuretic peptide (NT-proANP) and the N-terminal fragment of B-type natriuretic peptide (NT-proBNP) in severe trauma patients developing multiple organ dysfunction syndrome (MODS). Out of the 126 severe trauma patients that were admitted to the Emergency Intensive Care Unit of the General Hospital of Shenyang Military Region between January 2009 and December 2011, 26 patients with multiple injuries and an injury severity score (ISS) of $>16$ points were included in the study. The MODS score was calculated on admission as well as 24,48 and $72 \mathrm{~h}$ after the injury. Patients were divided into two groups: Group A consisted of patients with minor signs of organ dysfunction (MODS score, $\leq 4$ points) and group B of patients with major organ dysfunction (MODS score, $>4$ points). Venous blood $(5 \mathrm{ml})$ was extracted from the patients on admission and 6, 12, 24, 48 and $72 \mathrm{~h}$ after the injury. The Elecsys proBNP ${ }^{\circledR}$ and proANP ${ }^{\circledR}$ assays were used to determine the NT-proBNP and NT-proANP levels, respectively. The changes in the levels of C-reactive protein, white blood cells and neutrophils were detected and analyzed on admission. Acute physiology and chronic health evaluation II scores and ISSs were collected $72 \mathrm{~h}$ after the injury. The hemodynamic monitoring of cardiac index (CI) was performed using The Pulse index Continuous Cardiac Output system. The serum NT-proANP and NT-proBNP concentrations were elevated in all 26 patients. Upon admission, the serum NT-proANP and NT-proBNP values were 637.3 \pm 8.9 and $137.3 \pm 8.9 \mathrm{pmol} / \mathrm{l}$, respectively, in group $\mathrm{A}$ and 1,185.0 \pm 7.2 and $185.0 \pm 7.2 \mathrm{pmol} / \mathrm{l}$, respectively, in group B. The NT-proANP and NT-proBNP levels in group A were significantly lower than those in group B at all subsequent time-points $(\mathrm{P}<0.001)$. By contrast, the $\mathrm{CI}$ in
\end{abstract}

Correspondence to: Dr Yan Gao, Department of Emergency Medicine, General Hospital of Shenyang Military Region, 83 Wenhua Road, Shenyang, Liaoning 110016, P.R. China

E-mail: nanzhiwu@163.com

Key words: severe trauma, multiple organ dysfunction syndrome, NT-proANP, NT-proBNP, cardiac impairment group A was significantly higher than that in group B at all time-points $(\mathrm{P}<0.001)$. An inverse correlation was observed between the NT-proANP or NT-proBNP concentration and $\mathrm{CI}$ at 24, 48 and $72 \mathrm{~h}$ after the injury $(\mathrm{r}=-0.679$ and -0.772 , respectively; $\mathrm{P}<0.001)$. In conclusion, the serum NT-proANP and NT-proBNP concentrations following multiple injuries have been found to be significantly correlated with the clinical signs of MODS, and a distinct correlation has been observed between the levels of serums NT-proANP and NT-proBNP and decreased CI. The data of this pilot study suggest that NT-proANP and NT-proBNP levels may be of value in the diagnosis of post-traumatic cardiac impairment.

\section{Introduction}

Severe post-traumatic complications, such as pyohemia and systemic inflammatory response syndrome (SIRS), comprise the major cause of mortality in hospitals, with a mortality rate of 30-50\%. SIRS can eventually result in multiple organ dysfunction syndrome (MODS) (1). In the later stages of severe trauma, blood vessels throughout the body, but particularly small blood vessels, have a low responsiveness to vasoactive drugs. As a consequence, the blood pressure cannot be effectively improved; it becomes difficult to enhance tissue perfusion; cells become subject to oxygen deficit; the damage progressively worsens and the release of inflammatory factors is increased, leading to a 'waterfall effect'. These events comprise the major pathological factors of $\operatorname{MODS}(2,3)$.

A number of studies have shown that certain cytokines that are intimately associated with inflammation, such as endothelin-1 and Toll-like receptors, play an important role in the development of organ dysfunction (4,5); however, these results have only been evident in animal studies and therefore cannot be used to generate a clinical index for the diagnosis of MODS during its development in humans. Other studies have found that serum atrial natriuretic peptide (ANP) and $\beta$-type natriuretic peptide (BNP) levels exhibit marked increases in numerous types of disease, including heart failure, acute lung injury (ALI), septic shock, burns and electrical injury, and that these increases are closely associated with the hemodynamic index $(6,7)$. Compared with traditional inflammatory markers and trauma scoring systems, however, the correlation between the levels of serum N-terminal peptide of pro-ANP (NT-proANP) and N-terminal fragment of BNP (NT-proBNP), 
which are used as indicators of the trauma-induced development of MODS, and the hemodynamic index following severe trauma is rarely reported. The aim of the present study, therefore, was to evaluate the prognostic value of serum NT-proANP and NT-BNP in severe trauma patients with MODS and to perform a correlation analysis between these indicators and the hemodynamic index.

\section{Materials and methods}

Clinical data. One hundred and sixteen severe trauma patients, who had been admitted to the Emergency Intensive Care Unit of the General Hospital of Shenyang Military Region (Shenyang, China) between January 2009 and December 2011, were examined. All the necessary clinical tests were performed, including vital signs, blood, urine and liver and kidney function tests and blood gas analysis. In addition, all patients underwent central venous catheterization, Pulse index Continuous Cardiac Output (PiCCO) measurement (Pulsion Medical Systems SE, Munich, Germany), electrocardiography, blood pressure and heart rate monitoring, and blood oxygen saturation and blood gas analyses. The inclusion criteria were as follows: i) No injury history; ii) injury severity score (ISS), $\geq 16$; iii) age, $\geq 18$ years; iv) time interval between trauma and admission, $<90 \mathrm{~h}$. In addition, patients meeting the following criteria were excluded from the study: i) Mortality within $24 \mathrm{~h}$ after trauma; ii) main diagnosis, cardiac trauma; iii) intracranial hemorrhage; iv) heart, liver, kidney or endocrine history. Following admission, the age, gender, cause of trauma and medical history of the patients were recorded. The patients were given $24 \mathrm{~h}$ fluid resuscitation and anti-inflammatory treatment, and the aforementioned tests and observations, including 24-h urine volume and PiCCO monitoring, were performed. This study was approved by the ethics committee of the General Hospital of Shenyang Military Region, and informed consent was obtained from all patients or patient guardians.

Grouping method. The MODS score (Table I) was calculated on admission, as well as 24,48 and $72 \mathrm{~h}$ after the injury. The patients were divided into two groups: Group A (MODS scores $\leq 4$ points over 2 days) and group B (MODS scores $>4$ points).

Testing indices. Venous blood $(5 \mathrm{ml})$ was collected following admission and 6, 12, 24,48 and $72 \mathrm{~h}$ after the injury. Differences in serum levels of NT-proANP and NT-proBNP were detected using a rapid detector (Roche Diagnostics Gmbh, Mannheim, Germany). The normal serum NT-proANP and NT-proBNP levels are $<1,000$ and $<100 \mathrm{pmol} / 1$, respectively. The changes in the levels of C-reactive protein (CRP), white blood cells (WBCs) and neutrophils were detected and analyzed on admission (in $24 \mathrm{~h}$ ). The acute physiology and chronic health evaluation II (APACHE II) scores and ISSs were collected $72 \mathrm{~h}$ after the injury. The hemodynamic monitoring of the cardiac index (CI) was performed upon admission and 24, 48, and $72 \mathrm{~h}$ after the injury using the PiCCO system.

Statistical analysis. SPSS software (version 15.0; SPSS, Inc., Chicago, IL, USA) was used for the statistical analysis and the t-test was used for the comparison between the two groups. The results are presented as the mean \pm standard deviation, and $\mathrm{P}<0.05$ was considered to indicate a statistically significant difference. In the correlation analysis, $\mathrm{P}<0.05$ was considered to indicate a statistically significant linear correlation. The closer the absolute value of the correlation coefficient (r) to 1 , the stronger its relevance.

\section{Results}

Basic patient characteristics. Out of the 116 examined patients, 26 were included in the study; 16 were assigned to group A and 10 to group B. No significant differences were found in the basic characteristics and the ISSs between the two groups $(\mathrm{P}>0.05)$; by contrast, the APACHE II score of the patients in group B was significantly higher than that of the patients in group $\mathrm{A}(\mathrm{P}<0.05)$ (Table II).

MODS score. The MODS scores were calculated on admission, as well as 6, 12, 24, 48 and $72 \mathrm{~h}$ after the injury. The scores of the patients in group B were significantly higher than those of the patients in group A at the time of admission, as well as after 24, $48(\mathrm{P}<0.05)$ and $72 \mathrm{~h}(\mathrm{P}<0.01)$ (Table III).

Concentrations of serum NT-proANP and NT-proBNP. The concentrations of serum NT-proANP and NT-proBNP were measured on admission, as well as 6, 12, 24, 48 and $72 \mathrm{~h}$ after the injury. In group A, the concentrations of serum NT-proANP at the respective time-points were $637.3 \pm 8.9$, $857.4 \pm 5.8,874.1 \pm 8.6,999.3 \pm 11.0,1,024.9 \pm 15.6$ and $1,087.6 \pm 7.5 \mathrm{pmol} / 1$, while in group B they were 1,185.0 \pm 7.2 , $1,901.9 \pm 11.0,2,288.3 \pm 11.3,2,908.2 \pm 12.0,3,558.2 \pm 143.0$ and $4,295.9 \pm 160.8 \mathrm{pmol} / 1$. Twelve hours after admission, the difference in the serum NT-proANP concentration between the two groups was highly statistically significant $(\mathrm{P}<0.01)$ (Fig. 1).

The concentrations of serum NT-proBNP in group A on admission and at the 6-, 12-, 24-, 48- and 72-h time-points were $137.3 \pm 8.9,157.4 \pm 5.8,174.1 \pm 8.6,199.3 \pm 11.0,224.9 \pm 15.6$ and $191.6 \pm 7.5 \mathrm{pmol} / \mathrm{l}$, respectively, while in group B they were $185.0 \pm 7.2,201.9 \pm 11.0,328.3 \pm 11.3,708.2 \pm 12.7,1,258.2 \pm 143.0$ and $1,936.9 \pm 160.8 \mathrm{pmol} / 1$, respectively. At $24 \mathrm{~h}$, the difference in the serum NT-proBNP concentration between the two groups was highly statistically significant $(\mathrm{P}<0.01)$ (Fig. 2).

Correlation analysis between the serum NT-proANP and NT-proBNP concentrations and the hemodynamic index. Seventy-two hours after the injury, the correlations between the serum NT-proANP and NT-proBNP concentrations and the CI (Table IV) and systemic vascular resistance index (SVRI) were analyzed. The SVRI was determined directly using the PiCCO machine. An inverse correlation was observed between the serum NT-proANP/NT-proBNP concentration and CI ( $r=-0.679$ and -0.772 , respectively; $\mathrm{P}<0.001)$. The NT-proBNP level showed a stronger correlation with the CI than the NT-proANP level (Figs. 3 and 4).

\section{Discussion}

Trauma is considered by some to be one of the worst epidemics of the 20th century. The number of recorded trauma victims has been unprecedented and the subsequent costs severe (1). 
Table I. Multiple organ dysfunction syndrome scoring.

\begin{tabular}{lccccc}
\hline Body system & 0 & 1 & 2 & 3 & 4 \\
\hline Respiratory system $\left(\mathrm{PaO}_{2} / \mathrm{FiO}_{2}\right)$ & $>300$ & $226-300$ & $151-225$ & $76-150$ & $\leq 75$ \\
Kidney system $(\mathrm{Cr}, \mu \mathrm{mol} / \mathrm{l})$ & $\leq 100$ & $101-200$ & $201-350$ & $351-500$ & $>500$ \\
Liver system $($ bilirubin, $\mu \mathrm{mol} / \mathrm{l})$ & $\leq 20$ & $21-60$ & $61-120$ & $121-240$ & $>240$ \\
Cardiovascular system $\left(\mathrm{PAR}^{\mathrm{a}}\right)$ & $\leq 10.0$ & $10.1-15.0$ & $15.1-20.0$ & $20.1-30.0$ & $>30.0$ \\
Coagulation system $\left(\right.$ platelets, $\left.\mathrm{x} 10^{9} / \mathrm{l}\right)$ & $>120$ & $81-120$ & $51-80$ & $21-50$ & $\leq 20$ \\
Nervous system $(\mathrm{Glasgow}$ Coma Score $)$ & 15 & $13-14$ & $10-12$ & $7-9$ & $\leq 6$
\end{tabular}

${ }^{a} \mathrm{PAR}=$ heart rate $\mathrm{x}$ central venous pressure/mean arterial pressure. PAR, pulmonary arterial resistance; $\mathrm{PaO}_{2} / \mathrm{FiO}_{2}$, ratio of arterial oxygen partial pressure to fractional inspired oxygen; $\mathrm{Cr}$, creatinine.

Table II. Basic patient characteristics and clinical data $24 \mathrm{~h}$ after admission.

\begin{tabular}{lccc}
\hline $\begin{array}{l}\text { Patient } \\
\text { characteristics }\end{array}$ & $\begin{array}{c}\text { Group A, } \\
\mathrm{n}=16\end{array}$ & $\begin{array}{c}\text { Group B, } \\
\mathrm{n}=10\end{array}$ & P-value \\
\hline Males (\%) & 67.5 & 75.7 & $>0.05$ \\
Age (years) & $43 \pm 12.9$ & $51 \pm 14.3$ & $>0.05$ \\
ISS & $31.9 \pm 8.3$ & $39.2 \pm 10.1$ & $>0.05$ \\
APACHE II score & $21.8 \pm 3.1$ & $28.3 \pm 6.9$ & $<0.05$ \\
CRP (mg/l) & $107.7 \pm 18.7$ & $100.3 \pm 13.6$ & $>0.05$ \\
WBCs (x10 $/ 1)$ & $14.2 \pm 8.7$ & $12.2 \pm 10.0$ & $>0.05$ \\
Neutrophils $(\%)$ & $85.9 \pm 5.5$ & $80.9 \pm 7.1$ & $>0.05$
\end{tabular}

Results are presented as the mean \pm standard deviation. ISS, injury severity score; APACHE II, acute physiology and chronic health evaluation II; CRP, C-reactive protein; WBC, white blood cell.

Table III. Multiple organ dysfunction syndrome scores of the two groups.

\begin{tabular}{lccc}
\hline Time-point & Group A, $\mathrm{n}=16$ & Group B, $\mathrm{n}=10$ & P-value \\
\hline Admission & $3.1 \pm 0.6$ & $4.8 \pm 0.8$ & $<0.05$ \\
$24 \mathrm{~h}$ & $2.0 \pm 0.3$ & $5.1 \pm 0.4$ & $<0.05$ \\
$48 \mathrm{~h}$ & $1.8 \pm 0.3$ & $5.9 \pm 0.8$ & $<0.05$ \\
$72 \mathrm{~h}$ & $1.9 \pm 0.3$ & $7.0 \pm 0.8$ & $<0.01$ \\
\hline
\end{tabular}

Results are presented as the mean \pm standard deviation.

Despite the indisputable development of medical science, traumatic injuries and post-traumatic complications remain an important problem requiring an urgent solution.

Natriuretic peptides, including ANP, BNP and C-type natriuretic peptide, are cardiac hormones synthesized and secreted by ventricular myocytes. The endocrine activity of these hormones includes natriuresis, diuresis, dilation of blood vessels and inhibition of the renin-angiotensin-aldosterone and sympathetic nervous systems. The hormones are additionally known to play a positive role in the preload and afterload reduction in patients with heart failure (8). A previous study

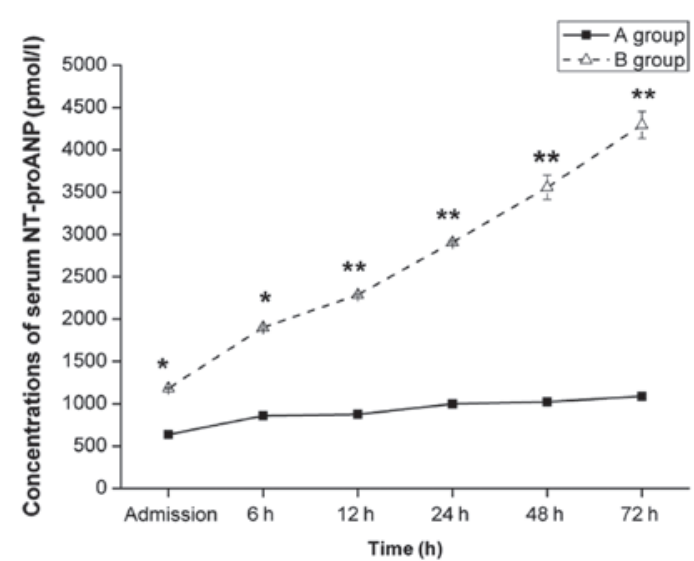

Figure 1. Concentration of serum NT-proANP at different time-points. ${ }^{*} \mathrm{P}<0.05$ and ${ }^{* *} \mathrm{P}<0.01$ vs. group A. NT-proANP, N-terminal peptide of pro-atrial natriuretic peptide.

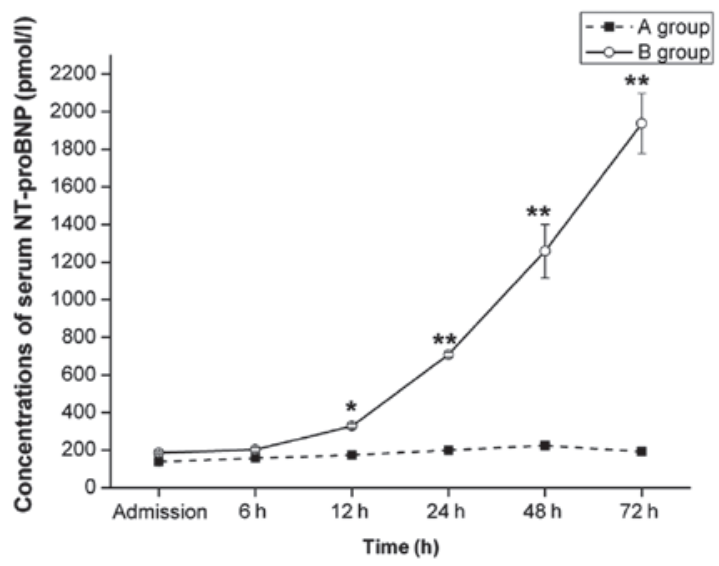

Figure 2. Concentration of serum NT-proBNP at different time-points." $\mathrm{P}<0.05$ and ${ }^{* *} \mathrm{P}<0.01$ vs. group A. NT-proBNP, N-terminal fragment of B-type natriuretic peptide.

has reported that the physiological effect of ANP is to control arterial blood pressure by regulating the CI and SVR, while BNP is involved in the acute inflammatory response to tissue damage (9). In severe post-traumatic complications, such as SIRS and MODS, the dysfunction in microcirculatory vasoconstriction causes a fall in blood pressure, which leads to 


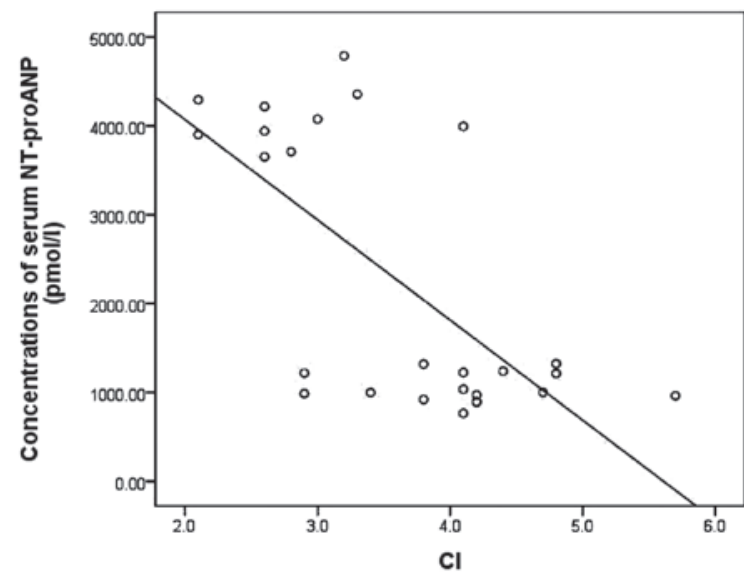

Figure 3. Linear correlation between serum NT-proANP concentration and the $\mathrm{CI} 72 \mathrm{~h}$ after the injury $(\mathrm{r}=-0.679, \mathrm{P}<0.001)$. NT-proANP, $\mathrm{N}$-terminal peptide of pro-atrial natriuretic peptide; $\mathrm{CI}$, cardiac index.

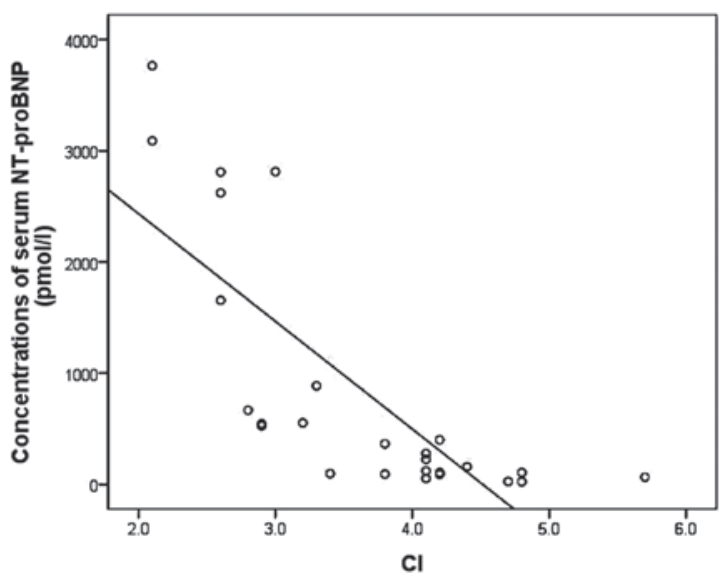

Figure 4. Linear correlation between serum NT-proBNP concentration and the $\mathrm{CI} 72 \mathrm{~h}$ after the injury $(\mathrm{r}=-0.772, \mathrm{P}<0.001)$. NT-proBNP, N-terminal fragment of B-type natriuretic peptide; CI, cardiac index.

Table IV. Cardiac index of the two groups.

\begin{tabular}{lcc}
\hline Time point & Group A $(\mathrm{n}=16)$ & Group B $(\mathrm{n}=10)$ \\
\hline $6 \mathrm{~h}$ & $4.0 \pm 1.1$ & $2.2 \pm 0.8$ \\
$12 \mathrm{~h}$ & $3.6 \pm 0.5$ & $2.0 \pm 0.5$ \\
$24 \mathrm{~h}$ & $4.0 \pm 0.9$ & $2.1 \pm 0.9$ \\
$48 \mathrm{~h}$ & $3.8 \pm 0.8$ & $1.9 \pm 1.1$ \\
$72 \mathrm{~h}$ & $4.1 \pm 0.7$ & $1.8 \pm 1.5$ \\
\hline
\end{tabular}

Results are presented as the mean \pm standard deviation. $\mathrm{P}<0.01$ for all time points in groups A and B.

little or no urine, dilation of the blood vessels and inhibition of the sympathetic nervous system, which negatively affects the state of the severe trauma patients (10).

Hoffmann and Brueckmann (11) measured the serum NT-proBNP level in 57 septic patients using ELISA and observed that the NT-proBNP concentration was notably increased compared with the levels of the controls. At an
NT-proBNP concentration of $>1,400 \mathrm{pmol} / 1$, a 3.9 -fold increase in mortality rate was found. NT-proBNP was therefore established as an indicator of the severity of sepsis (11). Kotanidou et al (12) studied 233 patients in a critical condition but with no heart disease and found that the serum NT-proBNP concentration increased as the condition of the patients was aggravated. A positive correlation was noted between the NT-proBNP concentration and inflammatory factors; therefore, the serum NT-proBNP concentration could be used independently to calculate the prognosis of critical patients. Mitaka et al (13) found that the serum NT-proBNP level in patients with ALI was positively correlated with the SVRI and pulmonary vascular resistance index and inversely correlated with the CI. A positive correlation between NT-proANP and pulmonary capillary wedge pressure showed that they had value for the diagnosis of ALI. The present study found that the NT-proANP and NT-proBNP levels of the 26 patients were higher than the normal values. The serum peptide concentrations in group B were significantly higher than those in group A and showed a sustained increase up to the 72-h time-point. Both indicators were inversely correlated with CI, which was consistent with the findings of previous studies (8).

Norepinephrine, a $\beta$-receptor agonist, is the first drug used to elevate blood pressure in cases of hypotension caused by traumatic hemorrhagic shock. Since $\beta$-receptor activation can increase the $\mathrm{CI}$ of severe trauma patients, the administration of norepinephrine may affect the left ventricular ejection fraction of the heart, altering left ventricular function. By contrast, the development of ALI and ARDS as a result of oxygen deficit following trauma leads to an increase in pulmonary vascular resistance, and the use of blood pressure-elevating drugs will further increase this resistance (8). The administration of catecholamine drugs therefore increases right ventricular afterload, as well as the concentrations of NT-proANP and NT-proBNP (14).

Although the ISS and APACHE II have been suggested to reflect the severity of the traumatic injuries and the physiological function following trauma, in addition to acting as predictors of post-traumatic complications and mortality, they have not, to date, been shown to be capable of predicting the occurrence of or enabling an early diagnosis of MODS. The results of the present study revealed that the basic characteristics and ISSs of the patients exhibited no significant differences; by contrast, the APACHE II scores in the two groups of patients were significantly different.

CRP, an acute-phase reactant synthesized in the liver in response to tissue injury or severe infection, is a marker of inflammation that is extensively used in the clinic. CRP has the ability to activate the complement system and improve the activity and movement of phagocytes. WBCs and neutrophils in the peripheral blood are indexes for the diagnosis of SIRS (15). It is generally recognized that severe trauma can produce an abundance of WBC chemotactic factors, resulting in increases in the number and activation of WBCs and the release of enzymes, such as elastase and matrix metalloproteinase. This leads to cell death, damage to the structural organization and organ dysfunction, and ultimately assists the development of MODS (16). It has been shown that, compared with CRP, the prognostic values of ANP and BNP in diagnosing and predicting the mortality of septic patients is 
greater (17). In the present study, no significant differences in the levels of CRP, WBCs or neutrophils were found between the two groups of patients exhibiting different severities of MODS; this suggested that the three indexes could not be used either to reflect the severity of MODS or as independent prognostic markers. These results were consistent with those of other studies (18).

In the past, body fluid states were monitored using urine volume and central venous pressure measurements or evaluations of hemodynamics using intrusive methods (Swan-Ganz method), leading to an increased risk of recurrent trauma and infection $(19,20)$. The present study determined the fluid load state of the body by performing continuous hemodynamic monitoring using the PiCCO system. Point-of-care testing is a relatively new concept in patient assessment. Point-of-care (or bedside) testing is defined as medical testing at or near the site of patient care. These are simple medical blood tests which may be performed at the bedside. Through the use of this rapid, simple, convenient and economic testing method, doctors can monitor the changes in serum NT-proANP and NT-proBNP levels in time and combine these changes with hemodynamic data in order to improve the diagnostic and therapeutic decisions for severe trauma patients.

In conclusion, this study has demonstrated that serum NT-proANP and NT-proBNP levels are closely correlated with the CI. Compared with other traditional inflammatory markers, NT-proANP and NT-proBNP are superior prognostic indicators for the development of MODS in severe trauma patients.

\section{References}

1. Brongel L: Guidelines for severe multiple and multiorgan traumatic injuries. Przegl Lek 60 (60 Suppl 7): 56-62, 2003 (In Polish).

2. Zweifach BW, Benacerraf B and Thomas L: The relationship between the vascular manifestations of shock produced by endotoxin, trauma, and hemorrhage. II. The possible role of the reticulo-endothelial system in resistance to each type of shock. J Exp Med 106: 403-414, 1957.

3. Zweifach BW and Thomas L: The relationship between the vascular manifestations of shock produced by endotoxin, trauma, and hemorrhage. I. Certain similarities between the reactions in normal and endotoxin-tolerant rats. J Exp Med 106: 385-401, 1957.
4. Freeman BD, Machado FS, Tanowitz HB and Desruisseaux MS: Endothelin-1 and its role in the pathogenesis of infectious diseases. Life Sci 118: 110-119, 2014.

5. Li P, Guo Y, Bledsoe G, Yang ZR, Fan H, Chao L and Chao J: Kallistatin treatment attenuates lethality and organ injury in mouse models of established sepsis. Crit Care 19: 200, 2015.

6. Determann RM, Royakkers AA, Schaefers J, de Boer AM, Binnekade JM, van Straalen JP and Schultz MJ: Serum levels of N-terminal proB-type natriuretic peptide in mechanically ventilated critically ill patients - Relation to tidal volume size and development of acute respiratory distress syndrome. BMC Pulm Med 13: 42, 2013.

7. Papanikolaou J, Makris D, Mpaka M, Palli E, Zygoulis P and Zakynthinos E: New insights into the mechanisms involved in B-type natriuretic peptide elevation and its prognostic value in septic patients. Crit Care 18: R94, 2014.

8. Chopra S, Cherian D, Verghese PP and Jacob JJ: Physiology and clinical significance of natriuretic hormones. Indian J Endocrinol Metab 17: 83-90, 2013.

9. Siemiatkowski A and Jabłonowska A: Natriuretic peptides - relevance in intensive care. Anestezjol Intens Ter 40: 96-102, 2008 (In Polish).

10. Roger F Shere-Wolfe, Samuel M Galvagno, Jr, Thomas E Grissom, et al: Critical care considerations in the management of the trauma patient following initial resuscitation. Scand J Trauma Resusc Emerg Med 20: 68, 2012.

11. Hoffmann U and Brueckmann M: A new language of natriuretic peptides in sepsis? Crit Care Med 36: 2686-2687, 2008.

12. Kotanidou A, Karsaliakos P, Tzanela M, et al: Prognostic importance of increased plasma amino-terminal pro-brain natriuretic peptide levels in a large noncardiac, general intensive care unit population. Shock 31: 342-347, 2009.

13. Mitaka C, Hirata Y, Nagura T, et al: Increased plasma concentrations of brain natriuretic peptide in patients with acute lung injury. J Crit Care 12: 66-71,1997.

14. Behnke BJ, Zawieja DC, Gashev AA, et al: Diminished mesenteric vaso- and venoconstriction and elevated plasma ANP and BNP with simulated microgravity. J Appl Physiol (1985) 104: 1273-1280, 2008.

15. Castelli GP, Pognani C, Cita M, et al: Procalcitonin, C-reactive protein, white blood cells and SOFA score in ICU: Diagnosis and monitoring of sepsis. Minerva Anestesiol 72: 69-80, 2006.

16. Barati M, Alinejad F, Bahar MA, et al: Comparison of WBC, ESR, CRP and PCT serum levels in septic and non-septic burn cases. Burns 34: 770-774, 2008.

17. Yucel T, Memiş D, Karamanlioglu B, et al: The prognostic value of atrial and brain natriuretic peptides, troponin I and C-reactive protein in patients with sepsis. Exp Clin Cardiol 13: 183-188, 2008.

18. Yucel T, Memis D, Karamanlioglu B, Süt N and Yuksel M The prognostic value of atrial and brain natriuretic peptides, troponin I and C-reactive protein in patients with sepsis. Exp Clin Cardiol 13: 183-188, 2008.

19. Konarzewski W: Pulmonary artery catheterisation. Pulmonary artery catheters should be banned from intensive care units. BMJ 313: 1328, 1996.

20. Simini B: Pulmonary artery catheters in intensive care. Lancet 366: 435-436, 2005. 